\title{
Perception fields: analysing distributions of optical features as a proximity navigation tool for autonomous probes around asteroids
}

\author{
Marco Zaccaria Di Fraia \\ Centre for Electronic Warfare \\ Information and Cyber \\ Cranfield University, The \\ Defence Academy Of The UK \\ Shrivenham, SN6 8LA, UK \\ marco.di-fraia@cranfield.ac.uk
}

\author{
Luke Feetham \\ Centre for Electronic Warfare \\ Information and Cyber \\ Cranfield University, The \\ Defence Academy Of The UK \\ Shrivenham, SN6 8LA, UK \\ 1.feetham@cranfield.ac.uk
}

\author{
Leonard Felicetti \\ Space Research Group, Centre of \\ Autonomous and Cyber-Physical \\ Systems \\ Cranfield University \\ MK43 0AL, Bedfordshire, UK \\ leonard.felicetti@cranfield.ac.uk
}

\author{
Joan-Pau Sanchez \\ Space Research Group, Centre of \\ Autonomous and Cyber-Physical \\ Systems \\ Cranfield University \\ MK43 0AL, Bedfordshire, UK \\ jp.sanchez@cranfield.ac.uk
}

\author{
Lounis Chermak \\ Centre for Electronic Warfare \\ Information and Cyber \\ Cranfield University, The \\ Defence Academy Of The UK \\ Shrivenham, SN6 8LA, UK \\ 1.chermak@cranfield.ac.uk
}

\begin{abstract}
This paper suggests a new way of interpreting visual information perceived by visible cameras in the proximity of small celestial bodies. At close ranges, camera-based perception processes generally rely on computational constructs known as features. Our hypothesis is that trends in the quantity of available optical features can be correlated to variations in the angular distance from the source of illumination. Indeed, the discussed approach is based on treating properties related to these detected optical features as readings of a field - the perception fields of the title, assumed induced by the coupling of the environmental conditions and the state of the sensing device. The extreme spectrum of shapes, surface properties and gravity fields of small celestial bodies heavily affects visual proximity operational procedures. Therefore, self-contained ancillary tools providing context and an evaluation of estimators' performance while using the least number of priors are extremely significant in these conditions. This preliminary study presents an analysis of the occurrences of optical feature observed around two asteroids, 101955 Bennu and (8567) 1996 HW1 in visual data simulated within Blender, a computer graphics engine. The comparison of three different feature detectors showed distinctive trends in the distribution of the detected optical features, directly correlated to the spacecrafttarget-Sun angle, confirming our hypothesis.
\end{abstract}

Keywords-Optical Navigation, Small Celestial Bodies, Space Systems, Perception, Feature Detection

\section{INTRODUCTION}

The first decades of landing operations in astronautics have been completely dominated by the selection of large gravity landing targets. However, after the Lunar Apollo missions, landing itself, for a while, appeared to become a mission type of minor relevance. It was only around the beginning of this millennium that this mission type started to become extremely significant again. The first time a synthetic device touched down on the surface of a body significant within the scope of this work, concerned with low gravity objects, was in 2001, when the probe NEAR Shoemaker [1] landed on 433 Eros, a Near Earth Asteroid (NEA). This was

Marco Zaccaria Di Fraia's PhD is sponsored by Thales Alenia Space rapidly followed by several others missions: the Hayabusa mission to the asteroid Itokawa in 2005 [2], the Philae lander of the Rosetta mission to the comet 67P/ChuryumovGerasimenko in 2014 [3], the Hayabusa2 mission to the asteroid Ryugu in 2018 [4], and more recently the OSIRISREx probe to the asteroid Bennu in 2020 [5]. Moreover, many more missions to similar small targets are expected to take place in the near future [6], [7]. The targets of these missions alone highlight a broad variability in the geological and morphological properties of these celestial objects, and they represent only a handful of the roughly 25000 Near Earth Asteroids [8] that are currently known.

We believe that one of the implications of this variety could be a paradigm shift in the development of proximity navigation processes. In particular, we expect future designs to be dominated by methods capitalizing on elements remaining consistent between targets and relying as little as possible on models and priors. Camera-driven navigation appears to be particularly suited for these missions, as it presents a solution with a minimal hardware/software cost profile and provides extensive additional information and situational awareness that can readily be exploited.

Previous optical navigation designs for proximity operations, have generally relied on prominent external structures and patterns, processed through one, or more, perception models [9]-[12]. The digital representation of these observables is called a feature, and the algorithms operating their search and selection deemed feature detectors. The detected features, then, generally require to be identified and matched across subsequent frames to return localisation information. The complex environment around asteroids is rather disruptive for this process. Features might not be stable enough under the complex illumination conditions, and their appearance could rapidly change as a result of the possibly fast, complex rotational dynamics. Moreover, large scale features like craters might be completely absent from asteroids [9]; therefore should not be targeted when designing general approaches. 
Following these premises, in this work we propose a counterintuitive approach which can be used to increase the robustness of feature-based methods, while providing an additional navigation tool in resource constrained conditions. Accordingly, bulk information on features is interpreted in a way that is analogous to readings of fields for physical quantities, like a magnetic or a gravitational field. These are the perception fields found in the title. These readings can be used either to structure ancillary navigation functions, or to validate the outputs of concurrent processes. The rationale behind this idea results from the fact that, while lighting can have deleterious effects on individual local structures by changing their appearance over time, it conversely induces seemingly regular distributions of collective feature properties. In particular, this paper focuses on demonstrating the correlation observed between the spacecraft-Sun-targetangle, or Sun phase angle $\varphi$, and curves generated by enumeration of available features per detector, among the selected ones. Specifically, we have tested two assumptions, fundamental to understand if there is the potential to integrate this information in navigation processes:

1. For a given set of fixed target conditions, properties associated with optical features are distributed as a function of the Sun phase angle.

2. Anomalies in this distribution are potentially helpful discriminants to resolve ambiguities.

\section{Simulation PARAMETERS}

The scope of the simulation process is to generate sets of visual data enabling the determination of the curves showing the quantitative distribution of features for each observing station. A code portion written in MATLAB 2019b [13] is used to define the positions and attitude of each camera; these are then transferred to Blender [14] through a .csv file to generate the outputs of the simulated camera.

We analyse circular sets of points around two simulated targets representing possible NEO targets, with different shapes and levels of surface resolution. These are a model of 101955 Bennu with a resolution of $75 \mathrm{~cm}$ [15] and a coarse model of HW1 generated from ground-based observations [16]. To avoid defining a lower observational limit considering the point where the size of voxels becomes comparable to the distance sampled on the ground for each pixel, we perform a smoothing of the models within Blender. This allows avoiding the emergence of spurious features induced by voxels occupying more than 1 pixel. In both cases the surface material was set to be a Diffuse BSDF with an RGB value of $R=15, G=15, B=15$ and a null roughness value. Within Blender the models have the dimension reported in Table I. The orientation of Bennu was left unchanged from that of the retrieved CAD model, while HW1 was rotated in order to have its longest axis aligned with Bennu's polar axis. This offered us the ability to equally configure distances, Sun orientation and camera models in both cases. The final output consisted of images as the ones illustrated in Fig. 1 and Fig. 2, all acquired at an equivalent angular station. Due to the lack of albedo or texture distributions we are only able to consider features generated from the interaction of structure and illumination. This choice is justified by the assumption that features induced by textural elements are uniformly distributed. Therefore, their effect on the shape of the illumination-related feature distribution curve would be limited to variations in amplitude.

All the test cases have been generated for static Sun conditions. The field labeled 'energy' of the simulated stellar light source, defining its intensity within Blender, is set to 5, so as to maintain a modestly powerful level of illumination. The acquisitions are performed at 7.5 times the average radius $R_{a}$ of Bennu, where $7.5 R_{a}=1860 \mathrm{~m}$, with an angular step of $2^{\circ}$, from $0^{\circ}$ to $358^{\circ}$, by center-pointing cameras moving in circles in the equatorial plane of the target asteroids. The simulated camera is based on the design of the GomSpace NanoCam C1U [17] assumed to have been equipped with a lens having a focal length of $35 \mathrm{~mm}$, and a focal plane of 2048 x 1536 pixels. Three feature detectors were employed as observables in this study: Harris-Stephens corners [18], Oriented FAST and rotated BRIEF (ORB) [19] and Speeded Up Robust Features (SURF) [20], which are well established in computer vison and aerospace domains.

TABLE I. TARGETS' DIMENSIONS

\begin{tabular}{|c|c|c|c|}
\cline { 2 - 4 } \multicolumn{1}{c|}{} & $\mathbf{X}$ & $\mathbf{Y}$ & $\mathbf{Z}$ \\
\hline Bennu & $563 \mathrm{~m}$ & $535 \mathrm{~m}$ & $497 \mathrm{~m}$ \\
\hline HW1 & $1241 \mathrm{~m}$ & $488 \mathrm{~m}$ & $535 \mathrm{~m}$ \\
\hline
\end{tabular}

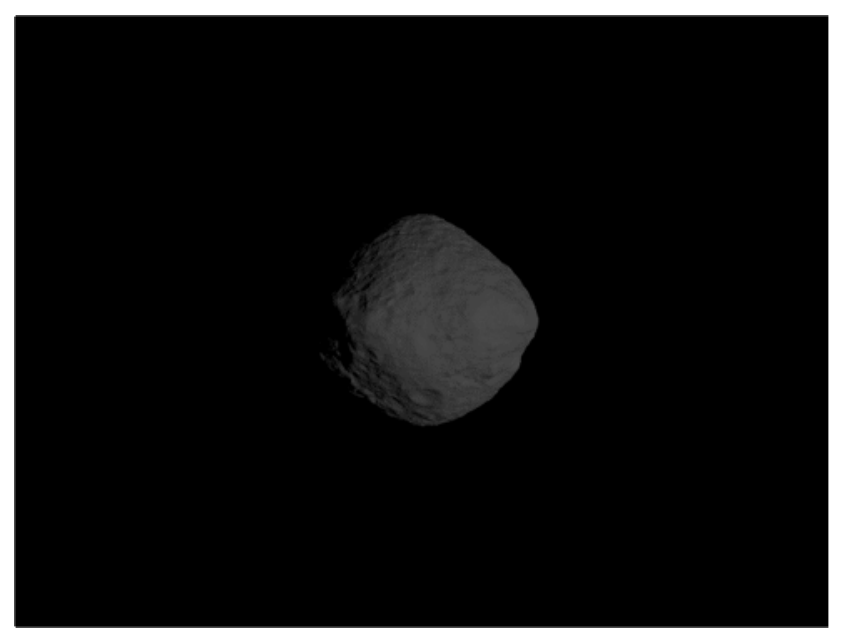

Fig. 1 -Simulated Bennu (Angular station: $324^{\circ}$ )

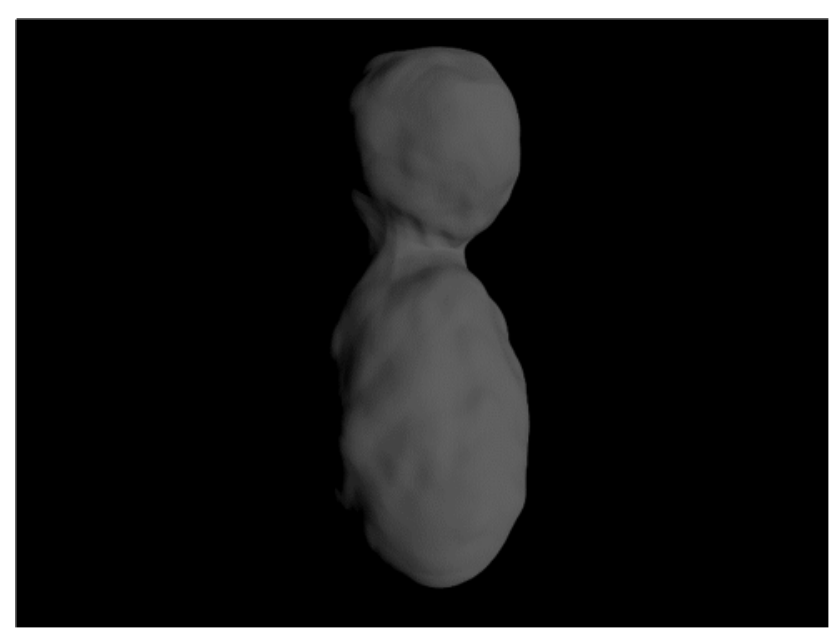

Fig. 2 - Simulated coarse HW1 (Angular station: 324²) 


\section{RESULTS}

This section presents the results of this study. The large number of parameters involved led to the choice of only two simple test cases which present preliminary results as a foundation for more ambitious future tests in more challenging configurations. Since at this stage our interest lies in the shape of these distributions, rather than their numeric values, all the curves are normalized with respect to their maximum value. The list of these maxima is reported in Table II. Future work will be focus on providing heuristic laws describing the distributions of these maxima (amplitude).

TABLE II. MAXIMUM NUMBER OF AVAILABLE FEATURES PER TEST CASE

\begin{tabular}{|c|c|c|c|c|}
\cline { 3 - 5 } \multicolumn{2}{c|}{} & \multicolumn{3}{c|}{ Maximum Number of Available Features } \\
\cline { 3 - 5 } \multicolumn{2}{c|}{} & Harris & ORB & SURF \\
\hline \multirow{2}{*}{$\begin{array}{c}\text { Test } \\
\text { Case }\end{array}$} & TC1 & 495 & 32 & 6 \\
\cline { 2 - 5 } & TC2 & 671 & 280 & 26 \\
\hline
\end{tabular}

In the first set of test conditions, labelled test case 1 (TC1), the target is HW1 and the Sun direction is set to be coincident with the direction of the $\mathrm{X}$ axis, coming from $+\mathrm{X}$. This configuration is shown in Fig. 4. For test case 2 (TC2) the Sun direction is rotated so as to arrive at an angle of $+120^{\circ}$ from the $+\mathrm{X}$ axis, and the target is chosen to be Bennu. This configuration is shown in Fig. 6. Hence, comparing TC1 and TC2 enables observing the effect of different targets, and the effect of a different Sun position. In both cases, the Sun direction is coplanar with the plane containing the motion.
Fig. 3 shows the distribution of features around HW1 (TC1). It is possible to observe that in the proximity of regions having a null solar phase angle, the normalised number of ORB features goes to zero due to the flattening effect of direct illumination, which suppresses distributed features from shading. On the other hand, the number of normalised Harris corners tends to increase. The opposite behaviour is present at $\pm 90^{\circ}$ from the Sun direction, where the number of Harris feature drops while that of ORB feature grows. Hence, once the amplitude is known, comparing these retrieved values allows the determination of a coarse estimate for the angular position of the probe. As it can be seen from Fig. 5, showing results from TC2, SURF features have a distribution similar to ORB ones. The effect observed in Fig. 5 around $\pm 90^{\circ}+$ $k 360^{\circ}$, where the normalised number of Harris features tends to low, almost constant, values, is generated by 'Optical attractors' (features with a high metric quality score) through a mechanism addressed in a previous paper [21]. MATLAB's Harris feature detector has an acceptance law such that, among all the found features, those with a metric score above that of the largest value multiplied by a constant $<1$ are selected as viable features. Driving up the largest value - i.e., observing one (or more) extremely good feature - means cutting a large number of lower quality features.

In Fig. 3 and Fig. 5 the Sun direction is shown as a shaded vertical region. Acquisitions performed at an angular distance from the Sun direction $>160^{\circ}$ will be neglected. This is because they are associated to a region extremely difficult to handle from the point of view of camera operations. This area too is shown as a differently coloured shaded vertical region. The abscissa of all the graphs ranges from 0 to 718 degrees, so as to show with clarity the behaviour around $0^{\circ}+k 360^{\circ}$.

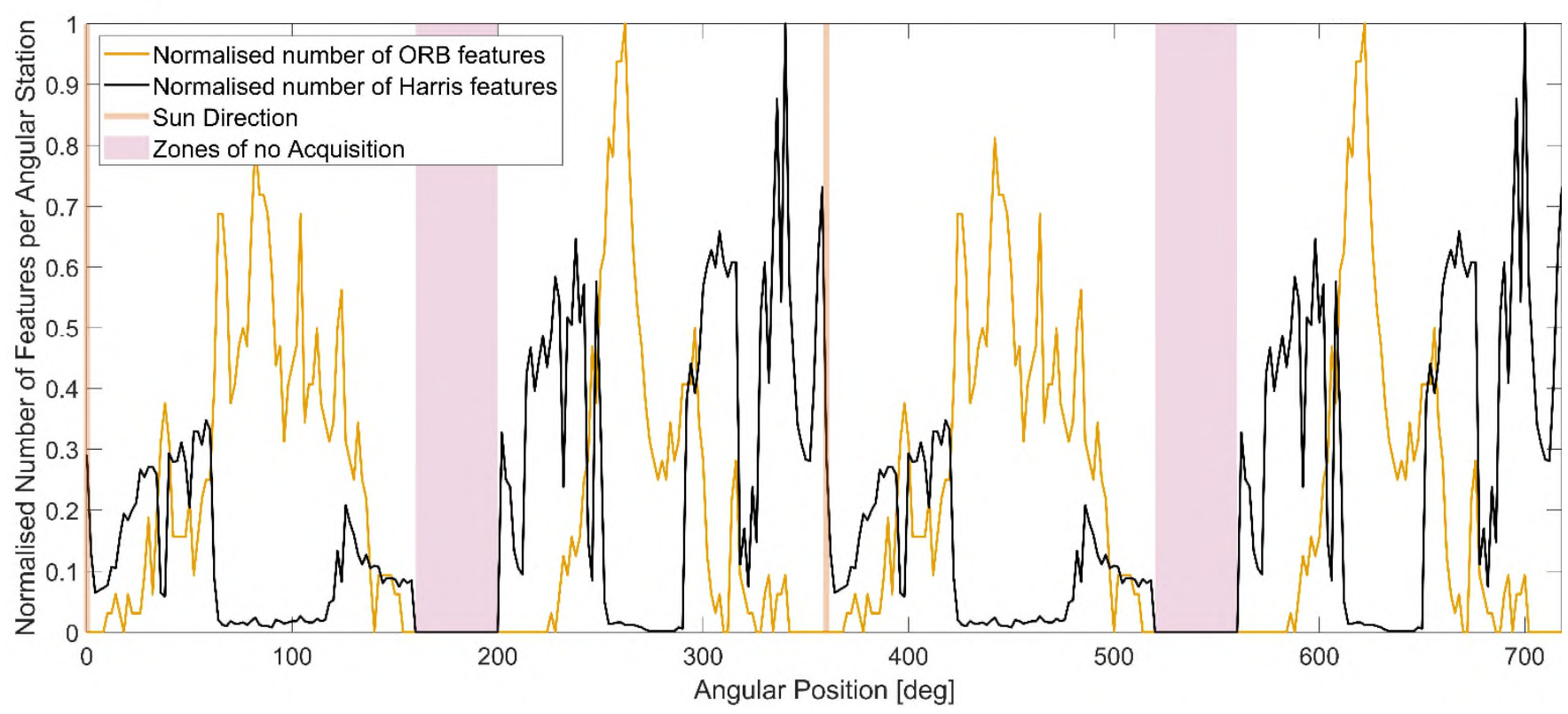

Fig. 3 - The distribution of features around 1996 HW1 with the Sun is arriving at $0^{\circ}$ from the + X direction (TC1). SURF Features were suppressed for clarity. 


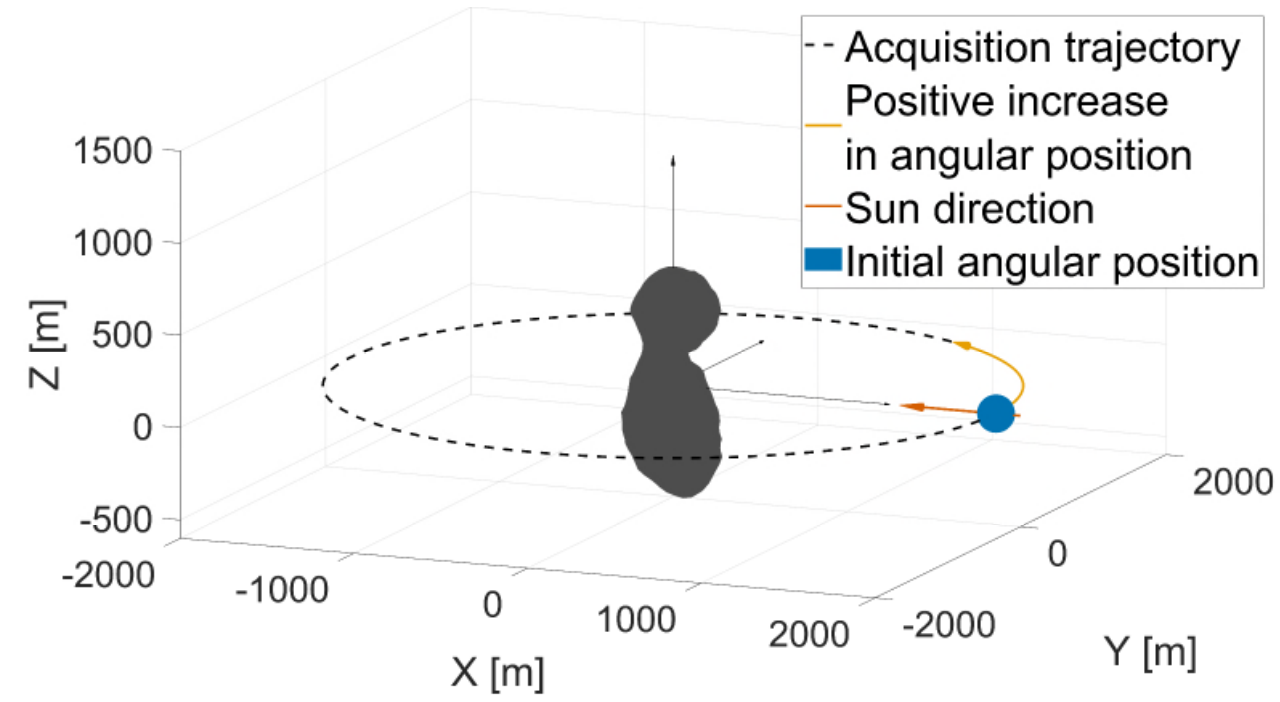

Fig.4 - Acquisition conditions for TC1. The dimensions of the targets are $\mathrm{X}=1241 \mathrm{~m} ; \mathrm{Y}=488 \mathrm{~m} ; \mathrm{Z}=535 \mathrm{~m}$.

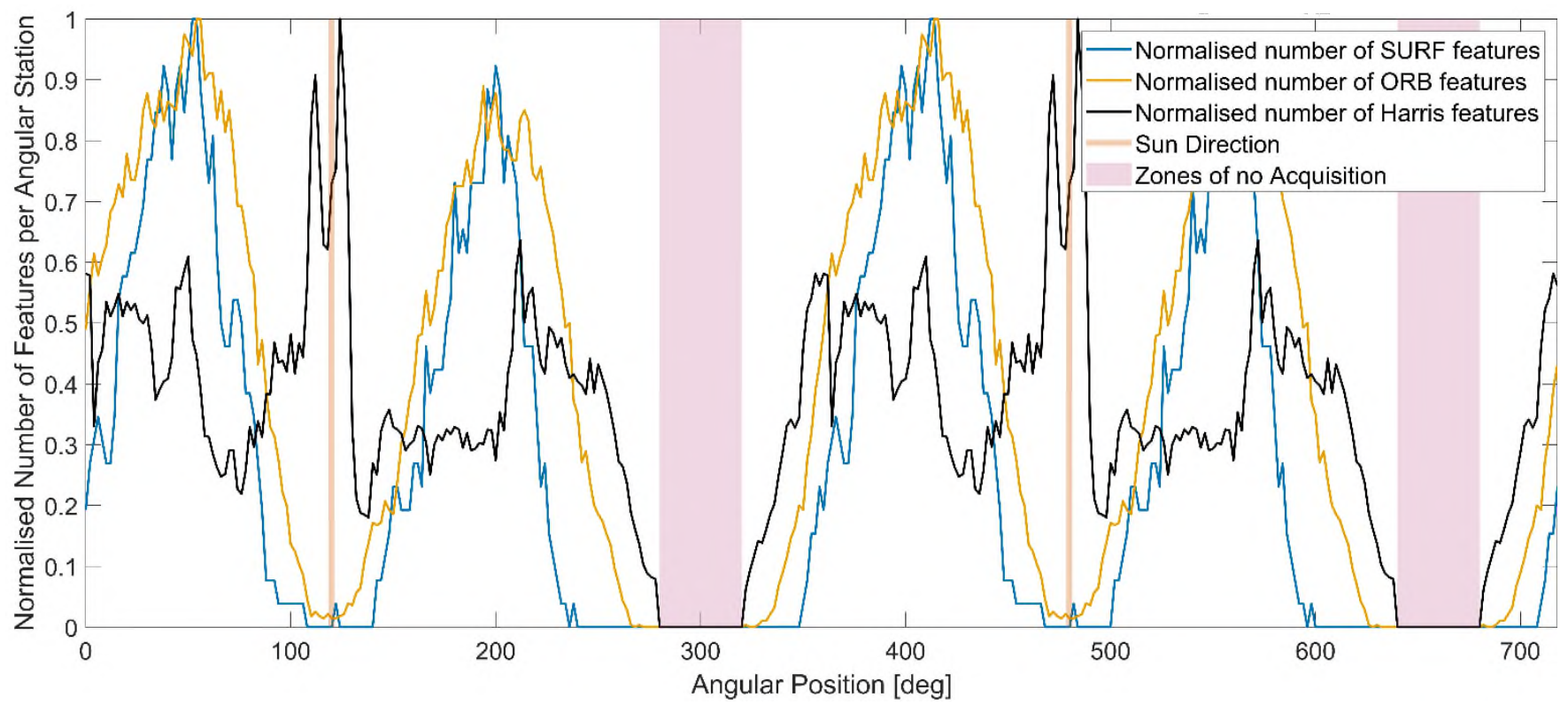

Fig. 5 - The distribution of features around Bennu with the Sun is arriving at $120^{\circ}$ from the $+\mathrm{X}$ direction (TC2).

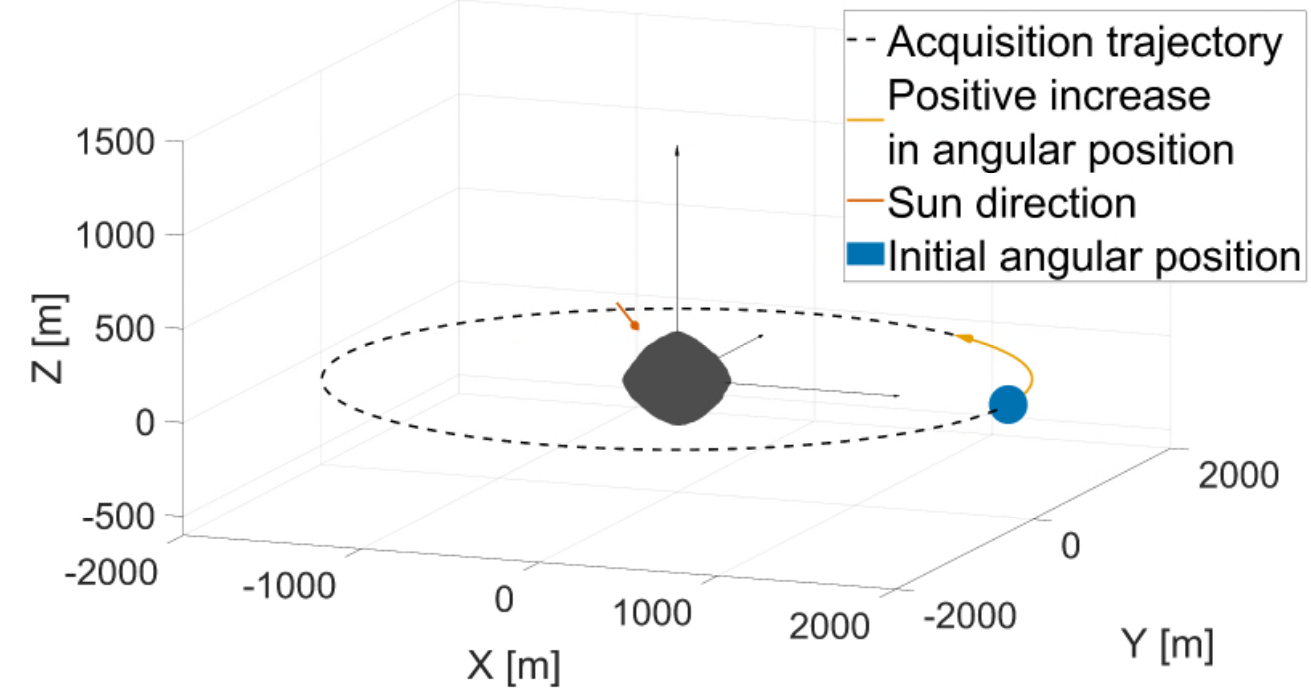

Fig. 6 - Acquisition conditions for TC2. The dimensions of the targets are $\mathrm{X}=563 \mathrm{~m} ; \mathrm{Y}=535 \mathrm{~m} ; \mathrm{Z}=497 \mathrm{~m}$. 


\section{CONCLUSIONS}

In this pilot study, we laid the groundwork for developing navigation strategies that rely on the distribution of visual features that are available in the proximity of small celestial bodies. Our initial results demonstrated that the quantity of structural features detected around targets of this type is not random, but rather, follows a distribution, primarily induced by the Sun phase angle, and only secondly by the local topology. This validates the hypothesis that sequential observations of the collective behaviour of features can be exploited to assist either in the retrieval of the probe's localisation information, or in the selection of an optimal detector. Therefore, it appears legitimate to further pursue the development of methods where these readings are used to complement or improve approaches considering features as distinct units requiring accurate labelling and tracking.

Future work will involve generating response maps using spherical acquisition patterns. This information could then be fed to machine learning algorithms, able to reconstruct the altitude and the pose of the sensor by comparing the number of available features, the number of available surface pixels and the ephemerides of the target. Ultimately, this would potentially enable a camera-only, target agnostic solution.

\section{REFERENCES}

[1] J. Veverka et al., "The landing of the NEAR-Shoemaker spacecraft on asteroid 433 Eros," Nature, 2001, doi: $10.1038 / 35096507$

[2] H. Yano et al., "Touchdown of the Hayabusa spacecraft at the muses sea on Itokawa," Science (80-. )., 2006, doi: 10.1126/science. 1126164

[3] H. Boehnhardt et al., "The Philae lander mission and science overview," Philosophical Transactions of the Royal Society A: Mathematical, Physical and Engineering Sciences. 2017. doi: 10.1098/rsta.2016.0248.

[4] Y. Tsuda, T. Saiki, F. Terui, S. Nakazawa, M. Yoshikawa, and S. ichiro Watanabe, "Hayabusa2 mission status: Landing, roving and cratering on asteroid Ryugu," Acta Astronaut., 2020, doi: 10.1016/j.actaastro.2020.02.035.

[5] D. S. Lauretta et al., "OSIRIS-REx: Sample Return from Asteroid (101955) Bennu," Space Science Reviews. 2017. doi: 10.1007/s11214-017-0405-1.

[6] M. A. Barucci et al., "Small D-type asteroids in the NEO population: New targets for space missions," Mon. Not. R. Astron. Soc., 2018, doi: 10.1093/mnras/sty532.

[7] L. McNutt, L. Johnson, P. Kahn, J. Castillo-Rogez, and A. Frick, "Near-Earth Asteroid (NEA) Scout," in AIAA SPACE 2014 Conference and Exposition, Reston, Virginia: American Institute of Aeronautics and Astronautics, Aug. 2014. doi: $10.2514 / 6.2014-4435$

"CNEOS - Discovery Statistics."
https://cneos.jpl.nasa.gov/stats/totals.html

C. Cocaud and T. Kubota, "SURF-based SLAM scheme using octree occupancy grid for autonomous landing on asteroids," Proc. 10th Int. Symp. Artif. Intell. Robot. Autom. Space., 2010.
Kawaguchi, "Optical navigation for asteroid explorer by use of virtual feature points," in AIAA/AAS Astrodynamics Specialist Conference, 2016, 2016. doi: 10.2514/6.2016-5213.

[11] S. Li, P. Cui, and H. Cui, "Autonomous navigation and guidance for landing on asteroids," Aerosp. Sci. Technol., 2006, doi: 10.1016/j.ast.2005.12.003.

[12] S. Bhaskaran, S. Nandi, S. Broschart, M. Wallace, L. Alberto Cangahuala, and C. Olson, "Small body landings using autonomous onboard optical navigation," in Journal of the Astronautical Sciences, 2011. doi: 10.1007/BF03321177.

[13] 2019 The Mathworks, Inc. MATLAB, Version 9.6, "MATLAB 2019b - MathWorks,"Www.Mathworks.Com/Products/Matlab. 2019.

[14] B. O. Community, "Blender - a 3D modelling and rendering package," Blender Foundation, 2020

[15] NASA/Goddard/University of Arizona, "Bennu shape model files." https://www.asteroidmission.org/updated-bennu-shapemodel-3d-files/

[16] C. Magri et al., "Radar and photometric observations and shape modeling of contact binary near-Earth Asteroid (8567) 1996 HW1," Icarus, 2011, doi: 10.1016/j.icarus.2011.02.019.

[17] "NanoCam C1U Datasheet", [Online]. Available: https://gomspace.com/UserFiles/Subsystems/datasheet/gs-dsnanocam-c1u-17.pdf

[18] C. Harris and M. Stephens, "A Combined Edge and Corner Detector," in Proceedings of the fourth Alvey Vision Conference (ACV88), 1988.

[19] E. Rublee, V. Rabaud, K. Konolige, and G. Bradski, "ORB: An efficient alternative to SIFT or SURF," in Proceedings of the IEEE International Conference on Computer Vision, 2011. doi: 10.1109/ICCV.2011.6126544.

[20] H. Bay, A. Ess, T. Tuytelaars, and L. Van Gool, "Speeded-Up Robust Features (SURF)," Comput. Vis. Image Underst., 2008, doi: 10.1016/j.cviu.2007.09.014.

[21] M. Z. Di Fraia and L. Chermak, "Countermeasure Leveraging Optical Attractor Kits (CLOAK): Interpretational disruption of a visual-based workflow," in Proceedings of SPIE - The International Society for Optical Engineering, vol. 11539, 2020. doi: $10.1117 / 12.2574099$ 